\title{
Design of Limb for Parallel Mechanism Based on Screw Theory*
}

\author{
Zhigang Lai ${ }^{1}$, Lixin $\mathrm{Li}^{2}$, and Ping' an $\mathrm{Liu}^{2}$ \\ ${ }^{1}$ Jiangxi Technical College of Manufacturing, Nanchang, Jiangxi, 330095, China \\ ${ }^{2}$ School of Mechanical and Electronic Engineering at East China Jiaotong University, \\ Nanchang 330013, China \\ lilixinsky@163.com
}

\begin{abstract}
Based on the reciprocal relationship of twist and wrench in screw theory, the mathematical model for limb of parallel manipulator is established in this paper. According to the motion modes of mobile platform (translation or rotation), we concluded the geometric conditions which the prismatic joint or revolute joint must meet with by analyzing the constraint screw on the platform, which provides the background for development of parallel mechanism.
\end{abstract}

Keywords: parallel mechanism; crew theory: limb; geometric condition.

\section{Introduction}

In recent year, since parallel mechanism can offer higher stiffness and larger load capability than those of serial mechanism, it has become a hot research topic in international robotics area. However, it is very difficult to design because of the complexity of kinematics and dynamics, the diversity of limb and the coupling of architecture.[1-3]

It is the most important task to meet with the DOF of the required motion for designing the parallel mechanism. In fact, DOF is the outward feature. The key is the design of constraint to implement the DOF of the required motion. The DOF of motion is objective in the limb. However, the constraint is designed in the limb by designer. And there are strict requirements to the geometric conditions which the prismatic joint or revolute joint must meet with in the limb.[4-5]

Based on the reciprocal relationship, in this paper, we concluded the geometric conditions which the prismatic joint or revolute joint must meet with in the limb by analyzing the constraint screw on the platform. According to the limb, we can design the parallel mechanism which is satisfied the required movement. It is a common method to the basic design of the parallel mechanism.

\section{Structural Synthesis of the Constraint Screw on the Platform}

Each limb should provide a constraint screw for the moving platform in the parallel mechanism. According to the constraint characteristics provided to the platform,

* Supported by Natural Science Foundation of Jiangxi, China, NO. 2008GZC005.

D. Li, Y. Liu, and Y. Chen (Eds.): CCTA 2010, Part I, IFIP AICT 344, pp. 508-518, 2011.

(C) IFIP International Federation for Information Processing 2011 
screw can be divided into twist and wrench. An arbitrary motion screw in space can include six motions at most, three translations along the $\mathrm{X}, \mathrm{Y}, \mathrm{Z}$ axes and three rotations around the $\mathrm{X}, \mathrm{Y}, \mathrm{Z}$ axes[6-8].

Twist should provide constraint for movement in space. It can be defined a $\$_{r}=\left(s_{r} ; r_{r} \times s_{r}\right)$. In the formula, $s_{r}$ stands for a unit vector along axis of twist and $r_{r}$ for a point on the axis direction of twist. Wrench should provide constraint for rotation in space. It can be defined as $\$_{r}=\left(\begin{array}{llllll}0 & 0 & 0 ; l_{r} & m_{r} & n_{r}\end{array}\right)$ and here we have $l_{r}^{2}+m_{r}^{2}+n_{r}^{2}=1$.

Twist and wrench are decided by the structural conditions of limb in parallel mechanism. So the type of constraint depends on the structural conditions of limb. According to the type of constraint, we can obtain the characteristic of limb structural. According to the difference of constraint in the limb, they can be divided into unconstrained limb, single constrained limb, double constrained limb, three-constrained limb, four-constrained limb, five-constrained limb and six-constrained limb. However, the five-constrained limb and six-constrained limb belong to planar limb which have no requirement in the space.

This paper analyzes the limb types including: the limb providing only one twist, the limb with two twists, the limb with three twists; the limb with only one wrench, the limb with two wrenches, the limb with three wrenches; the limb with one twist and one wrench, the limb with two twists and one wrench, the limb with one twist and two wrenches.

\subsection{The Limb with Only One Twist}

The basic expression of screw is $\mathscr{S}_{r}=\left(s_{r} ; r_{r} \times s_{r}\right)$. It is known from the reciprocal of screw that the limb should consist of the five independent screws which are reciprocal with the twist .

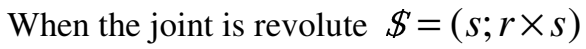

$$
\not{ }_{r} \circ \not{S}=s_{r} \bullet(r \times s)+s \bullet\left(r_{r} \times s_{r}\right)=s_{r} \bullet\left[\left(r-r_{r}\right) \times s\right]=0
$$

According to the geometric feature of vector, it should be known that there is a common perpendicular among $s_{r}, r-r_{r}$ and $S$, that is to say, the axis direction of the revolute joint and the axis direction of twist must be in the same plane.

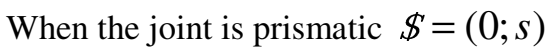

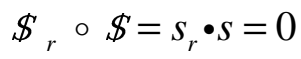

According to the geometric feature of vector, it should be known that $S_{r}$ and $S$ must be perpendicular each other, that is to say, the moving direction of the prismatic joint and the axis direction of twist must be perpendicular each other.

When the structural conditions of limb meet with the above requirements, the limb should provide only one twist. According to the requirements above, we should put up the limb-RRPRR shown as Fig. 1. First, we construct two parallel revolute joints, 
which make sure the direction of twist. Second, we establish the moving direction of the prismatic joint and the axis direction of twist must be vertical each other. Last, the axis direction of the last two revolute joints must intersect at o point which is the point of action of $s_{r}$.

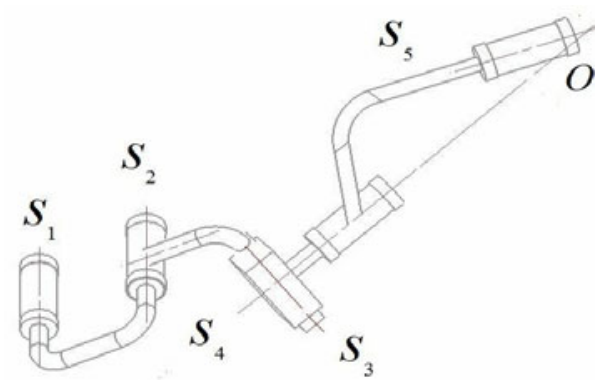

Fig. 1. Structur of Limb RRPRR-1F $\left(s_{r} / / s_{l} / / s_{2} \perp s_{3}, s_{4}\right.$ and $s_{5}$ intersect at $o, s_{r}$ acting on $o$ )

\subsection{The Limb with Two Twists}

The basic expression of screw is $\left\{\begin{array}{l}\$_{r 1}=\left(s_{r 1} ; r_{r 1} \times s_{r 1}\right) \\ \$_{r 2}=\left(s_{r 2} ; r_{r 2} \times s_{r 2}\right)\end{array}\right.$. It is known from the reciprocal of screw that the limb should consist of the four independent screws which are reciprocal with the two twists.

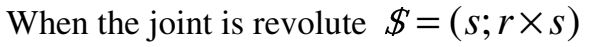

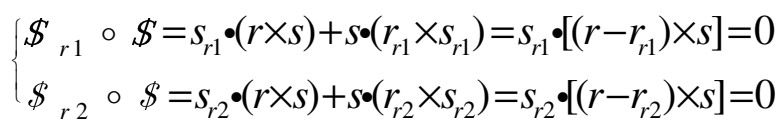

According to the geometric feature of vector, it is known that there is a common perpendicular among $S_{r 1}, r-r_{r 1}$ and $S$; a common perpendicular among $S_{r 2}$, $r-r_{r 2}$ and $S$, that is to say, the axis direction of the revolute joint must pass the point which is the point of intersection with the two twists or be parallel the plane which makes sure by the two twists.

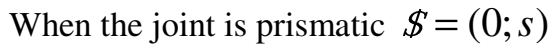

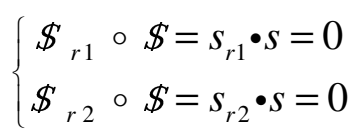

According to the geometric feature of vector, it should be known that $S_{r 1}$ and $S$ must be vertical each other; $S_{r 2}$ and $S$ must be vertical each other, that is to say, the moving direction of the prismatic joint must be parallel the cross-produce of the two twists. And there is the only prismatic joint in the limb. 
When the structural conditions of limb meet with the above requirements, the limb should provide two twists. According to the requirements above, we should put up the limb-RRPR shown as Fig. 2.

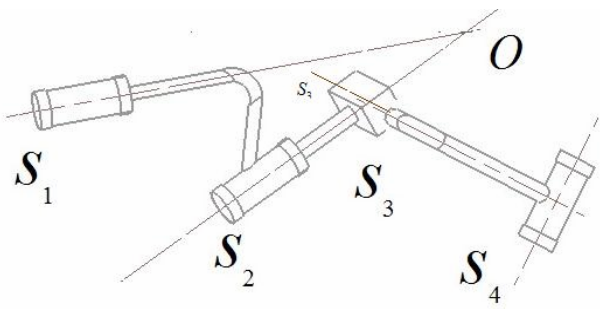

Fig 2. Structur of Limb RRPR-2F $\left(\mathrm{s} 1 \perp_{\mathrm{sr} 1 \times \mathrm{sr} 2 \mathrm{~s} 2} \perp_{\mathrm{sr} 1 \times \mathrm{sr} 2}\right.$, o is the point of action with sr1 and sr2)

\subsection{The Limb with Three Twists}

The basic expression of screw is

$$
\left\{\begin{array}{l}
\$_{r 1}=\left(s_{r 1} ; r_{r 1} \times s_{r 1}\right) \\
\$_{r 2}=\left(s_{r 2} ; r_{r 2} \times s_{r 2}\right) \\
\$_{r 3}=\left(s_{r 3} ; r_{r 3} \times s_{r 3}\right)
\end{array}\right.
$$

The limb should provide three independence twists which intersected at a point for platform. The limb constrained the three directions moving of the platform, that is to say, the platform just can be round the point to revolve. The limb should consist of three revolute joints, and that, there is only one type of the limb-RRR. According to the requirements above, we should put up the limb-RRR shown as Fig. 3.

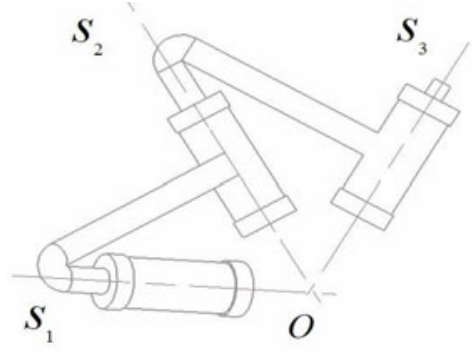

Fig. 3. Structur of Limb RRR-3F ( $s_{1}, s_{2}$, and $s_{3}$ intersect at $o$ )

\subsection{The Limb with Only One Wrench}

The basic expression of screw is $\$_{r}=\left(0 ; s_{r}\right)$. It is known from the reciprocal of screw that the limb should consist of the five independent screws which are reciprocal with the wrench. 


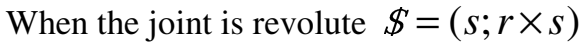

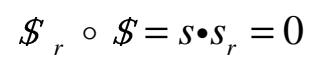

According to the geometric feature of vector, it should be known that $s_{r}$ and $s$ must be vertical each other, that is to say, the axis direction of the revolute joint and the axis direction of wrench must be vertical each other.

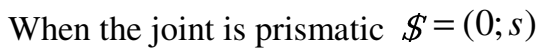

$$
\$_{r} \circ \$=0
$$

The equation is satisfied under any conditions, that is to say, the moving direction of the prismatic joint is independent of the axis direction of wrench.

When the structural conditions of limb meet with the requirements above, the limb should provide only one wrench. According to the requirements above, we should put up the limb-RRPRP show as Fig. 4.

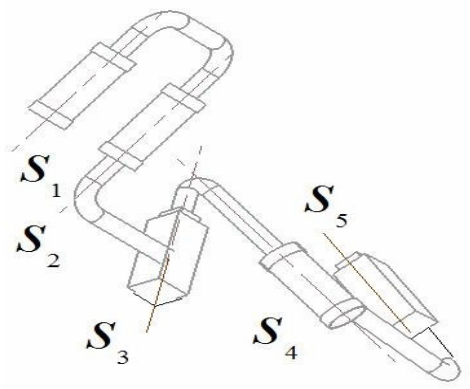

Fig. 4. Structur of Limb RRPRP-1M $\left(s_{1} / / s_{2}, s_{r} / / s_{2} \times s_{4}\right)$

\subsection{The Limb with Two Wrenches}

The basic expression of screw is $\left\{\begin{array}{l}\phi_{r 1}=\left(0 ; s_{r 1}\right) \\ \$_{r 2}=\left(0 ; s_{r 2}\right)\end{array}\right.$. It is known from the reciprocal of screw that the limb should consist of the four independent screws which are reciprocal with the two wrenches.

When the joint is revolute $\mathbb{S}=(s ; r \times s)$

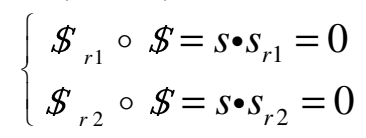

According to the geometric feature of vector, it should be known that $s_{r 1}$ and $s$ must be vertical each other; $s_{r 2}$ and $s$ must be vertical each other, that is to say, the axis direction of the revolute joint must be parallel the cross-produce of the two wrenches.

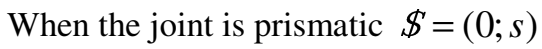

$$
\left\{\begin{array}{l}
\${ }_{r 1}^{\circ} \$=0 \\
\$_{r 2} \circ \$=0
\end{array}\right.
$$


The equation is satisfied under any conditions, that is to say, the moving direction of the prismatic joint is independent of the axis direction of the wrenches.

When the structural conditions of limb meet with the requirements above, the limb should provide only one wrench. According to the requirements above, we should put up the limb-RRPR shown as Fig. 5.

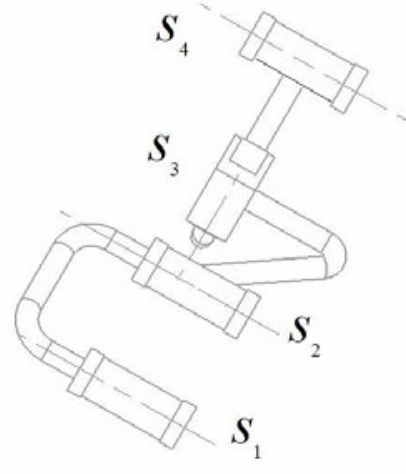

Fig. 5. Structur of Limb RRPR-2M $\left(s_{r 1} \times s_{r 2} / / s_{1} / / s_{2} / / s_{3}\right)$

\subsection{The Limb with Three Wrenches}

The basic expression of screw is

$$
\left\{\begin{array}{l}
\$_{r 1}=\left(0 ; s_{r 1}\right) \\
\$_{r 2}=\left(0 ; s_{r 2}\right) \\
\$_{r 3}=\left(0 ; s_{r 3}\right)
\end{array}\right.
$$

The limb should provide three independence wrenches which are independent each other. The limb constrained the three directions rotation of the platform, that is to say, the platform just can move along the X, Y, Z axis. The limb should consist of three independent prismatic joints, and that, there is only one type of the limb-PPP shown as Fig. 6.

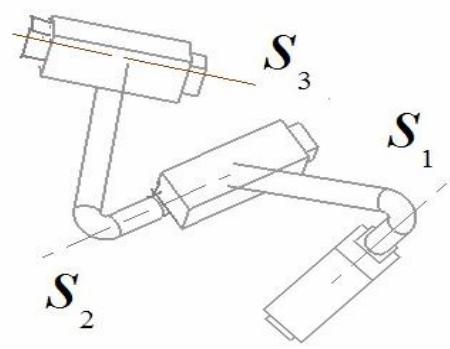

Fig. 6. Structur of Limb PPP-3M $\left(s_{1}, s_{2}\right.$ and $s_{3}$ are independent each other) 


\subsection{The Limb with One Twist and One Wrench}

The basic expression of screw is $\left\{\begin{array}{l}\$_{r 1}=\left(s_{r 1} ; r_{r 1} \times s_{r 1}\right) \\ \$_{r 2}=\left(0 ; s_{r 2}\right)\end{array}\right.$. It is known from the reciprocal of screw that the limb should consist of the four independent screws which are reciprocal with the one twist and one wrench.

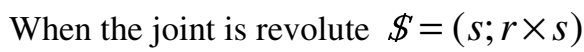

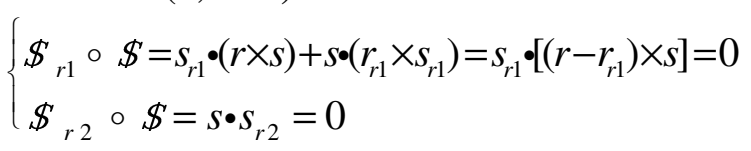

According to the geometric feature of vector, it should be known that there is a common perpendicular among $S_{r 1}, r-r_{r 1}$ and $S ; S_{r 2}$ and $S$ must be vertical each other, that is to say, the axis direction of the revolute joint must be located in the normal plane, which contained the twist, of the wrench.

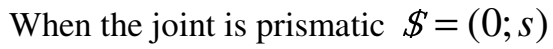

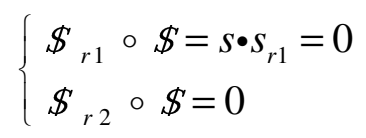

According to the geometric feature of vector, it should be known that $S_{r 1}$ and $S$ must be vertical each other, that is to say, the moving direction of the prismatic joint and the axis direction of twist must be vertical each other.

When the structural conditions of limb meet with the above requirements, the limb should provide one twist and one wrench. According to the requirements above, we should put up the limb-RPRR shown as Fig. 7.

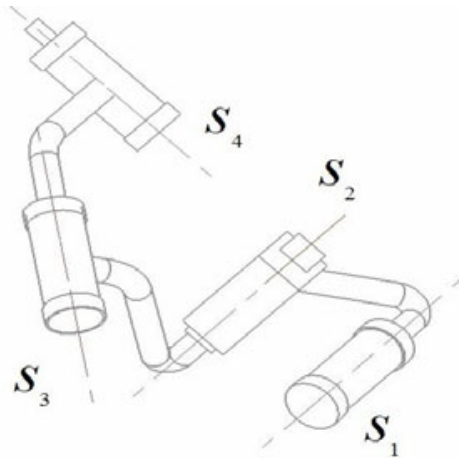

Fig. 7. Structur of Limb RPRR-1F1M $\left(s_{2} \perp_{s_{r 1}, s_{1}} \perp_{s_{r 2}}, s_{3} \perp_{s_{r 2}, s_{4}} \perp_{s_{r 2}, s_{1}, s_{3}, s_{4}}\right.$ and $s_{r 2}$ are in the same plane) 


\subsection{The Limb with Two Twists and One Wrench}

The basic expression of screw is

$$
\left\{\begin{array}{l}
\$ r 1=\left(s_{r 1} ; r_{r 1} \times s_{r 1}\right) \\
\$_{r 2}=\left(s_{r 2} ; r_{r 2} \times s_{r 2}\right) \\
\$_{r 3}=\left(0 ; s_{r 3}\right)
\end{array}\right.
$$

It is known from the reciprocal of screw that the limb should consist of the three independent screws which are reciprocal with the screws.

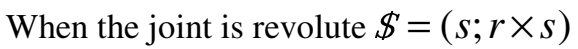

$$
\left\{\begin{array}{l}
\$_{r 1} \circ \$=S_{r 1} \bullet(r \times s)+s \bullet\left(r_{r 1} \times s_{r 1}\right)=S_{r 1} \bullet\left[\left(r-r_{r 1}\right) \times s\right]=0 \\
\$_{r 2} \circ \$=S_{r 2} \bullet(r \times s)+s \bullet\left(r_{r 2} \times s_{r 2}\right)=S_{r 2} \bullet\left[\left(r-r_{r 2}\right) \times s\right]=0 \\
\$_{r 3} \circ \$=s \bullet S_{r 3}=0
\end{array}\right.
$$

According to the geometric feature of vector, it should be known that there is a common perpendicular among $s_{r 1}, r-r_{r 1}$ and $S$; a common perpendicular among $s_{r 2}, r-r_{r 2}$ and $S ; s_{r 3}$ and $S$ must be vertical each other, that is to say, the axis direction of revolute joint, which must be vertical the axis direction of the wrench, must pass the point which is the point of intersection with the two twists or be parallel the plane which make sure by the two twists.

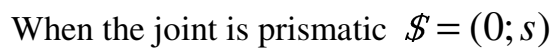

$$
\left\{\begin{array}{l}
\$_{r 1} \circ \$=s \bullet S_{r 1}=0 \\
\$_{r 2} \circ \$=s \bullet S_{r 2}=0 \\
\$_{r 3} \circ \$=0
\end{array}\right.
$$

According to the geometric feature of vector, it should be known that $S_{r 1}$ and $S$ must be vertical each other; $S_{r 2}$ and $S$ must be vertical each other; $S_{r 3}$ is independent of $S$, that is to say, the axis moving direction of the prismatic joint, which is independent of the axis direction of the wrench, must be parallel the cross-produce of the two twists.

When the structural conditions of limb meet with the above requirements, this limb should provide two twists and one wrench. According to the requirements above, we should put up the limb-RRP shown as Fig. 8. 


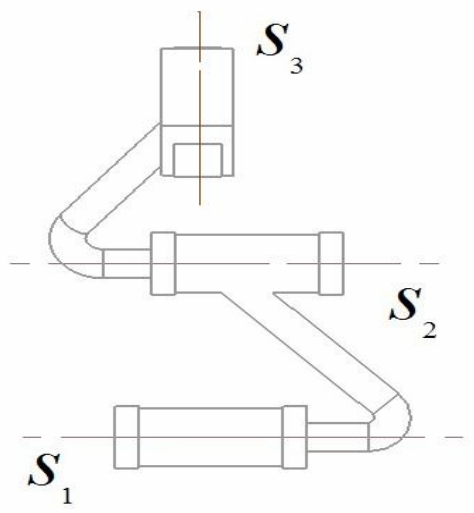

Fig. 8. Structur of Limb RRP-2F1M $\left(s_{1} \perp_{S_{r 3}, s_{2}} \perp_{s_{r 3}, s_{3}} \perp_{S_{r 1}, s_{3}} \perp_{s_{r 2}, s_{1}} \perp_{S_{r 1} \times s_{r 2}, s_{2}} \perp_{s_{r 1} \times s_{r 2}}\right)$

\subsection{The Limb with One Twist and Two Wrenches}

The basic expression of screw is

$$
\left\{\begin{array}{l}
\$_{r 1}=\left(s_{r 1} ; r \times s_{r 1}\right) \\
\$_{r 2}=\left(0 ; s_{r 2}\right) \\
\$_{r 3}=\left(0 ; s_{r 3}\right)
\end{array}\right.
$$

It is known from the reciprocal of screw that the limb should consist of the three independent screws which are reciprocal with the screws.

When the joint is revolute $\mathscr{S}=(s ; r \times s)$

$$
\left\{\begin{array}{l}
\$_{r 1} \circ \$=S_{r 1} \bullet(r \times s)+s \bullet\left(r_{r 1} \times s_{r 1}\right)=S_{r 1} \bullet\left[\left(r-r_{r 1}\right) \times s\right]=0 \\
\$_{r 2} \circ \$=s \bullet S_{r 2}=0 \\
\$_{r 3} \circ \$=s \bullet S_{r 3}=0
\end{array}\right.
$$

According to the geometric feature of vector, it should be known that there is a common perpendicular among $s_{r 1}, r-r_{r 1}$ and $S ; S_{r 2}$ and $S$ must be vertical each other; $S_{r 3}$ and $S$ must be vertical each other, that is to say, the axis direction of revolute joint, which is in the same plane with the twist, must be parallel the cross-produce of the two wrenches.

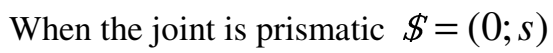

$$
\left\{\begin{array}{l}
\$_{r 1} \circ \not{S}=S \bullet S_{r 1}=0 \\
\$_{r 2} \circ \not{S}=0 \\
\$_{r 3} \circ \not{S}=0
\end{array}\right.
$$


According to the geometric feature of vector, it should be known that $S_{r 1}$ and $S$ must be vertical each other, that is to say, the axis moving direction of the prismatic joint must be vertical the axis direction of the twist.

When the structural conditions of limb meet with the above requirements, the limb should provide one twist and two wrenches. According to the requirements above, we should put up the limb-RRP shown as Fig. 9.

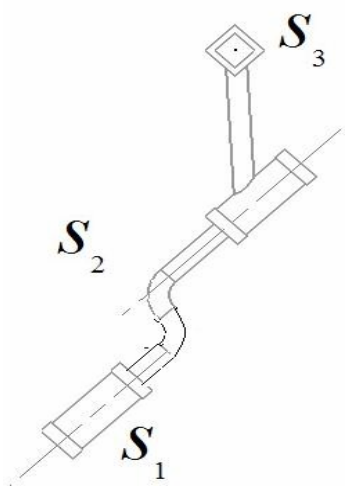

Fig. 9. Structur of Limb RRP-1F2M $\left(s_{1} / / s_{2} / / s_{r 2} \times s_{r 3}, s_{3} \perp_{s_{r l}}, s_{1}, s_{2}\right.$ and $s_{r 1}$ are in the same plane )

\section{Example of the Application}

Since it is regular for the geometric conditions of the joint in the limb, which connects fixed platform with mobile platform, the limb, which meets with the geometric conditions, can be used to establish the parallel mechanism which be satisfied with the mode of motion. Now take the limb Fig. 7 as an example to establish the 3-2T1R parallel mechanism as shown in Fig. 10. The limb in Fig. 7 provides one twist along the Z-axis, which constrain the moving along the Z-axis, and one wrench along the $\mathrm{X}$ axis, which constrain the rotation around the $\mathrm{X}$-axis. Thanks to the wrenches which provided by the three limbs are dependent in the XY plane, it should constrain the

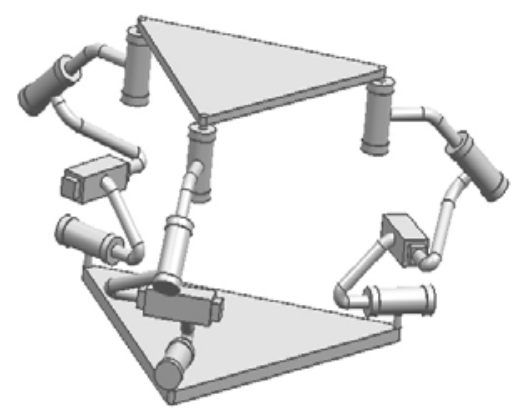

Fig. 10. 3-2T1R parallel mechanism 
rotation around the $\mathrm{X}$-axis and $\mathrm{Y}$-axis, that is to say, the platform just can revolve around $\mathrm{Z}$-axis and move in the XY plane.

\section{Conclusions}

According to analyze the type of limb and the type of restraint in screw theory, it is given a general design method of limb in the parallel mechanism. And that, it obtained the geometric conditions which the prismatic joint or revolute joint of over-constrained parallel mechanism must be meet with. The method analyzed from basic concept of the reciprocal produce in screw theory. It should make sure be general and pragmatic. It is a common reference value to the basic design of the parallel mechanism.

\section{References}

1. Ball, R.S.: A Treatise on the Theory of Screws. Cambridge University Press, Cambridge (1900)

2. Fang, Y., Tsai, L.-W.: Structure Synthesis of a Class of 4-DoF and 5-DoF Parallel Manipulators with Identical Limb Structures. The International Journal of Robotics Research 21(9), 799-810 (2002)

3. Herve, J.M., Sparacino, F.: Structural synthesis of "Parallel" Robots Generating Spatial Translation. IEEE, Los Alamitos (1991) 7803-0078/91/0600-0808\$01.00

4. Wang, J., Gosselin, C.M.: Kinematic Analysis and Singularity Loci of Spatial FourDegree-of-Freedom Parallel Manipulators Using a Vector Formulation. ASME Transactions, Journal of Mechanical Design 120(4), 555-558 (1988)

5. Tsai, L.-W.: Systematic Enumeration of Parallel Manipulators. Technical Research Report, T.R. $98-33$

6. Herve, J.M., Karoutia, M.: The Novel 3 - RUU Wrist with No Idle Pair. In: Proceedings of the Work-shop on Fundamental Issues and Future Research Directions for Parallel Mechanisms and Manipulators, Quebec, Canada, pp. 284-286 (2002)

7. Kong, X., Gosselin, C.M.: Type Synthesis of Three - Degree - of - Freedom Spherical Parallel Manipulators. The International Journal of Robotics Research 23(3), 237-245 (2004)

8. Karouia, M., Herve, J.M.: Non - over-constrained 3 DOF Spherical Parallel Manipulators of Type; 3 -RCC, 3 - CCR, 3 - CRC. Robotica 24, 85-94 (2006) 